\title{
INEQUALITIES FOR THE EIGENVALUES OF POWERS OF FUNCTIONS
}

\author{
DALLAS O. BANKS
}

In this paper, we give generalizations of a result of A. M. Fink. Specifically, we consider the Sturm-Liouville system

$$
\begin{aligned}
& \left(p(x) u^{\prime}\right)^{\prime}+\lambda \rho(x) u=0, \quad x \in(0, l), \\
& p(0) u^{\prime}(0)-h_{0} u(0)=0, \quad p(l) u^{\prime}(l)+h_{1} u(l)=0,
\end{aligned}
$$

where $p, \rho$ are integrable functions on $[0, l]$ with $p^{\prime}$ continuous and $\rho$ positive in a subinterval of $[0, l]$. Furthermore, $h_{0}, h_{1}$ are nonnegative numbers with $h_{0}, h_{1}$ approaching $\infty$ corresponding to the boundary conditions $u(0)=0, u(l)=0$, respectively. We will be concerned with the dependence of the positive eigenvalues of the system (1), (2) on the function $\rho$. We denote them accordingly by $0<\lambda_{1}[\rho]<\lambda_{2}[\rho]<\cdots$. Fink shows that in the case where (1), (2) reduces to

$$
\begin{aligned}
u^{\prime \prime}+\lambda \rho(x) u & =0, \quad x \in[0, l], \\
u(0) & =u(l)=0,
\end{aligned}
$$

the inequality

$$
\lambda_{1}\left[\rho^{2}\right] \leqq\left(l \lambda_{1}[\rho] / \pi\right)^{2}
$$

holds [1]. We prove the following

THEOREM 1. The positive eigenvalues of (1), (2) satisfy the functional inequality

$$
\lambda_{m+n-1}[\rho] \geqq\left(\lambda_{m}\left[\left|\rho_{1}\right|^{\alpha}\right]\right)^{1 / \alpha}\left(\lambda_{n}\left[\left|\rho_{2}\right|^{\beta}\right]\right)^{1 / \beta}
$$

where $\rho=\rho_{1} \cdot \rho_{2}$ and $1 / \alpha+1 / \beta=1$.

When $\rho_{2} \equiv 1$ on $[0, l], n=m=1, k=1, \alpha=\beta=2$, (4) reduces to Fink's inequality (3). Furthermore, the proof given here generalizes trivially to higher dimensional problems.

Proof of theOREM. We make use of the Rayleigh quotient

$$
R[\rho, u]=\frac{\int_{0}^{l} u^{\prime 2} d x+h_{0} u^{2}(0)+h_{1} u^{2}(l)}{\int_{0}^{l} \rho u^{2} d x}
$$

Received by the editors March 28, 1969. 
and the max-min characterization of the eigenvalues, i.e.,

$$
\lambda_{n+1}[\rho]=\max _{\left[v_{1}, \ldots, v_{n}\right]} d\left[v_{1}, \cdots, v_{n}\right],
$$

where $\left[v_{1}, \cdots, v_{n}\right]$ is the subspace spanned by $n$ smooth functions $v_{1}, \cdots, v_{n}$ and

$$
d\left[v_{1}, \cdots, v_{n}\right]=\min R[\rho, u],
$$

where the minimum is taken over all smooth functions satisfying the conditions

$$
\left(u, v_{k}\right)=\int_{0}^{l} \rho u v_{k} d x=0
$$

$(k=1, \cdots, n)$ and $\int_{0}^{l} \rho u^{2} d x \geqq 0$ (see [2, p. 214]). By Hölder's inequality, we have

$$
\begin{aligned}
\int_{0}^{l} \rho u^{2} d x & =\int_{0}^{l}\left(\rho_{1} u^{2 / \alpha}\right)\left(\rho_{2} u^{2 / \beta}\right) d x \\
& \leqq\left(\int_{0}^{l}\left|\rho_{1}\right|^{\alpha} u^{2} d x\right)^{1 / \alpha}\left(\int_{0}^{l}\left|\rho_{2}\right|^{\beta} u^{2} d x\right)^{1 / \beta}
\end{aligned}
$$

with $1 / \alpha+1 / \beta=1$.

Let $U=\left[u_{1}, u_{2}, \cdots, u_{m-1}\right]$ where $\left\{u_{k}\right\}_{k=1}^{m-1}$ is the set of eigenfunctions corresponding to $\left\{\lambda_{k}\left[\left|\rho_{1}\right|\right]\right\}_{k=1}^{n-1}$ and let $V=\left[v_{1}, v_{2}, \cdots, v_{n-1}\right]$ where $\left\{v_{k}\right\}_{k=1}^{n-1}$ is the set corresponding to $\left\{\lambda_{k}\left[\left|\rho_{2}\right|\right]\right\}_{k=1}^{n-1}$.

If $W=\left[u_{1}, \cdots, u_{m-1}, v_{1}, \cdots, v_{n-1}\right]$, then $(6)$ and $(7)$ yield

$$
\lambda_{m+n-1}[\rho] \geqq \min R\left[\rho_{1} u\right],
$$

where the minimum is taken over the set $\left\{u: u \in C^{\prime}, u \perp W, \int_{0}^{l} \rho u^{2} d x \geqq 0\right\}$. $\mathrm{By}^{\prime \prime}(8)$ and another application of the max-min principle we have

$$
\begin{aligned}
\lambda_{m+n-1}[\rho] & \geqq \min _{u \perp W}\left(R\left[\left|\rho_{1}\right|^{\alpha}, u\right]\right)^{1 / \alpha}\left(R\left[\left|\rho_{2}\right|^{\beta}, u\right]\right)^{1 / \beta} \\
& \geqq\left(\min _{u \perp U} R\left[\left|\rho_{1}\right|^{\alpha}, u\right]\right)^{1 / \alpha}\left(\min _{u \perp V} R\left[\left|\rho_{2}\right|^{\beta}, u\right]\right)^{1 / \beta} \\
& =\left(\lambda_{m-1}\left[\left|\rho_{1}\right| \alpha\right]\right)^{1 / \alpha}\left(\lambda_{n-1}\left[\left|\rho_{2}\right|^{\beta}\right]\right)^{1 / \beta} .
\end{aligned}
$$

Noting that the positive eigenvalues of (1), (2) are also functionals of $p$ and denoting them by $\lambda_{1}[p]<\lambda_{2}[p]<\cdots$, we are able to prove the following result.

THEOREM 2. The positive eigenvalues of (1) with boundary conditions $u(0)=u(l)=0$ and $\rho(x)>0, x \in[0, l]$ satisfy the inequality 


$$
\lambda_{m+n-1}[p] \geqq\left(\lambda_{m-1}\left[p_{1}^{\alpha}\right]\right)^{1 / \alpha}\left(\lambda_{n-1}\left[p_{2}^{\beta}\right]\right)^{1 / \beta}
$$

where $p=p_{1} \cdot p_{2}, p_{1}>0, p_{2}>0$ on $[0, l]$ and $1 / \alpha+1 / \beta=1$.

Proof. We note that $u_{\nu}$ is an eigenfunction of (1), (2) corresponding to the eigenvalue $\lambda_{\nu}[p]$ if and only if $u_{\nu}=p u_{\nu}^{\prime}$ is an eigenfunction of the reciprocal system

$$
\left(\frac{1}{\rho(x)} v^{\prime}\right)^{\prime}+\lambda \frac{1}{p(x)} v=0, \quad v^{\prime}(0)=v^{\prime}(l)=0
$$

corresponding to the eigenvalue $\lambda_{\nu}[p]$. We note, however, that the system (9) has zero for an eigenvalue corresponding to the eigenfunction $u_{0}(x)=$ const. The nonzero eigenvalues of (9) satisfy the following maximum-minimum principle:

Let $D$ denote the space of absolutely continuous functions such that

$$
\int_{0}^{l} \frac{1}{p} v u_{0} d x=\int_{0}^{l} \frac{1}{p} v d x=0 .
$$

Let $V_{n-1}$ denote the subspace of $D$ spanned by the set $\left\{v_{1}, v_{2}, \cdots\right.$, $\left.v_{n-1}\right\}$ taken from $D$. Denote the Rayleigh quotient corresponding to (9) by

$$
R[p, u]=\frac{\int_{0}^{l} \frac{1}{\rho} u^{\prime 2} d x}{\int_{0}^{l} \frac{1}{p} u^{2} d x}
$$

Then $\lambda_{n}[p]=\max _{V_{n-1}} \min _{u \perp V_{n-1}, u \in D} R[p, u]$.

The proof now follows as in the proof of Theorem 1.

Finally, we note that the results presented here are related to those in [3] where generalized means and in particular geometric means are discussed. Also, see reference [4].

\section{REFERENCES}

1. A. M. Fink, Eigenvalues of the square of a function, Proc. Amer. Math. Soc. 20 (1969), 73-74.

2. E. Kamke, Differentialgleichungen, Lösungsmethoden und Lösungen, Chelsea, New York, 1948.

3. D. O. Banks, Generalized means of eigenvalues, J. Math. Anal. Appl. 23 (1968), 409-420.

4. L. Janoš, Functional properties of the spectrum of boundary value problems, C̆asopis Pěst. Mat. 23 (1960), 461-474.

University of California, Davis 